\title{
Article \\ Development of A Nano-Apatite Based Composite Sealer for Endodontic Root Canal Filling
}

\author{
Angelica Bertacci $^{1, *}$, Daniele Moro ${ }^{2} \mathbb{D}$, Gianfranco Ulian ${ }^{2}\left(\mathbb{D}\right.$ and Giovanni Valdrè ${ }^{2, *}$ \\ 1 Department of Biomedical and Neuromotor Sciences (DiBiNeM), School of Dentistry, University of Bologna, \\ 40125 Bologna, Italy \\ 2 Department of Biological, Geological, and Environmental Sciences, University of Bologna, \\ 40126 Bologna, Italy; daniele.moro@unibo.it (D.M.); gianfranco.ulian2@unibo.it (G.U.) \\ * Correspondence: angelica.bertacci3@unibo.it (A.B.); giovanni.valdre@unibo.it (G.V.); \\ Tel.: +39-051-208-8111 (A.B.); +39-051-209-4943 (G.V.)
}

check for updates

Citation: Bertacci, A.; Moro, D.; Ulian, G.; Valdrè, G. Development of A Nano-Apatite Based Composite Sealer for Endodontic Root Canal Filling. J. Compos. Sci. 2021, 5, 30. https://doi.org/10.3390/jcs5010030

Received: 16 December 2020 Accepted: 14 January 2021 Published: 16 January 2021

Publisher's Note: MDPI stays neutral with regard to jurisdictional clai$\mathrm{ms}$ in published maps and institutional affiliations.

Copyright: (C) 2021 by the authors. Licensee MDPI, Basel, Switzerland. This article is an open access article distributed under the terms and conditions of the Creative Commons Attribution (CC BY) license (https:// creativecommons.org/licenses/by/ $4.0 /)$.

\begin{abstract}
Recently, endodontic sealers based on injectable bioactive materials were proposed to improve the filling of anatomical irregularities during root canal obturation. In this context, this preliminary work investigated the possibility of realizing a new calcium phosphate-based composite sealer for root canal filling with an optimized composition on setting kinetics and dentin tubules occlusion. Several calcium phosphate/liquid phase mixtures were initially evaluated for their workability, finding two suitable formulations. Both of them contained $66 \mathrm{wt} . \%$ of a nano-apatitebased cement (solid powdered phase). The liquid phase (34 wt.\%) comprised $13.6 \%$ propanediol and 20.4\% PEG 1000 (formulation 1), and formulation 2 comprised 27.2\% glycerin and 6.8\% PEG 200 (formulation 2). Then, these formulations were tested by means of permeability measurements and observation by scanning electron microscopy of treated model dentin samples. Both formulations succeeded in occluding dentinal tubules: the first one was able to create a full-bodied layer on dentin surface and, moreover, to resist, at least to a large extent, against citric acid attack. The second one showed a lower effectiveness after citric acid exposure. The composite compound that better satisfied the overall required characteristics of use, workability and sealing capacity was formulation 1 .
\end{abstract}

Keywords: nano-apatite; calcium phosphate; dentin permeability; scanning electron microscopy

\section{Introduction}

The main goal of root canal obturation is to provide an appropriate filling of anatomical irregularities with minimal voids [1,2]. To achieve this objective, gutta-percha cones are associated with endodontic sealers that work as sealing agents in filling root canals [2-4]. A variety of endodontic sealers is available, including zinc oxide eugenol, calcium hydroxide, glass ionomer, silicone, resin, and bioceramic-based sealers [4]. The latter kind of sealers includes alumina, zirconia, bioactive glass, glass ceramics, hydroxyapatite, and calcium phosphates [4]. Bioceramic-based sealers are categorized into two groups, the first one of sealers based on calcium silicate (Mineral Trioxide Aggregate (MTA)-based and nonMTA-based) and the second one based on calcium phosphate [5]. In addition, another categorization of bioceramic-based sealers is available in two groups of bioactive and bioinert materials due to their interaction with the close, alive tissues [6]. Bioactive materials, such as glass and calcium phosphate, interact with the surrounding tissue to encourage the growth of more durable tissues, exploiting their better biocompatibility and mechanical properties [7-10]. This implies releasing calcium ions, electroconductivity, production of calcium hydroxide, formation of an interfacial layer between the cement and dentinal wall and formation of apatite crystals over the surface of the material in a synthetic tissue fluid environment, such as phosphate buffer saline [4,11-13]. Bioceramic materials have a shorter setting time and a uniform consistency during placement, which improves handling with respect to white MTA [14]. In addition, cement mixed with water has a low cohesion before 
setting, leading to potential washout of the cement, and this could be avoided by using a premixed paste [15]. A premixed cement would simplify the dentist's work and ensure a precise, repeatable, liquid-to-powder ratio. A new generation of endodontic sealers has been produced based on calcium silicate as Total Fill BC Sealer (FKG Dentaire SA, La Chaux-de-Fonds, Switzerland) [4,16]. This sealer, also containing low amounts of calcium phosphate, is presented in a premixed syringe with an intracanal tip designed to be used with a single-cone filling technique [1,2].

The aim of this study is to develop a bioactive composite sealer for root canal filling based on an injectable calcium phosphate (nano-apatite), with adequate chemical and physical properties (setting time). The nano-apatite biomaterial was developed by the Interdisciplinary Research Centre of Biomineralogy, Crystallography and Biomaterials of the University of Bologna, Italy [17-19]. To assess the desired properties of the carrier, several composites with different ingredients that can be mixed with the calcium phosphate cement have been preliminarily prepared and evaluated for their workability. Then, the composite formulations with suitable properties were tested for their sealing capability by means of permeability measurements and observation by Scanning Electron Microscopy (SEM) of treated model dentin samples.

\section{Materials and Methods}

\subsection{Experimental Mixtures}

All the samples were prepared by weighing the materials with Gibertini E42S balance (Gibertini, Novate Milanese, Italy). They were then placed on ThermoScientific slides $(76 \mathrm{~mm} \times 26 \mathrm{~mm})$ using Carlo Erba wax paper sheets (Carlo Erba, Cornaredo, Italy). For the addition of liquid materials, Eppendorf Research pipettes (Eppendorf, Milano, Italy) were used.

The substances employed to create the carrier mixture are reported in Table 1.

Table 1. Experimental moisture ingredients.

\begin{tabular}{|c|c|}
\hline Substance & Description \\
\hline $\begin{array}{c}\text { PEG } 1000 \\
\text { Sigma-Aldrich, Merck Life Science S.r.l., Milano, Italy }\end{array}$ & $\begin{array}{l}\text { Polyethylene Glycol } 1000 \text { is a polymer obtained by polymerization } \\
\text { from ethylene oxide. It has a similar appearance to condensed milk and } \\
\text { is white, odourless and has a pH that varies between } 5 \text { and } 7 \text {. }\end{array}$ \\
\hline $\begin{array}{c}\text { PEG } 400 \\
\text { Sigma-Aldrich, Merck Life Science S.r.l., Milano, Italy }\end{array}$ & $\begin{array}{l}\text { Polyethylene Glycol } 400 \text { is a polymer obtained by polymerization from } \\
\text { ethylene oxide. It is a transparent and odourless liquid, with a pH that } \\
\text { varies between } 5 \text { and } 7 \text {. }\end{array}$ \\
\hline $\begin{array}{c}\text { PEG } 200 \\
\text { Sigma-Aldrich, Merck Life Science S.r.l., Milano, Italy }\end{array}$ & $\begin{array}{l}\text { Polyethylene Glycol 200, similarly to the previous one, is a polymer } \\
\text { obtained by polymerization from ethylene oxide. It is a transparent and } \\
\text { odourless liquid, with a pH that varies between } 5 \text { and } 7 \text {. }\end{array}$ \\
\hline $\begin{array}{c}\text { CORN OIL } \\
\text { Acros Organics, Rodano, Milano, Italy }\end{array}$ & $\begin{array}{l}\text { Corn oil is the common oil extracted from the germ contained in the } \\
\text { kernels of corn seeds (Zea Mays), a plant belonging to the Graminaceae } \\
\text { family. It is used not only in the kitchen, but also in the cosmetic field. }\end{array}$ \\
\hline $\begin{array}{l}\text { GLYCERINE } \\
\text { Glycerol RPE, Carlo Erba Reagents, } \\
\text { Cornaredo, Milano, Italy }\end{array}$ & $\begin{array}{c}\text { Glycerine, or glycerol, is an organic compound with three hydroxyl } \\
\text { groups, the presence of which makes it soluble in water. At room } \\
\text { temperature, it appears as a thick, viscous, and sweetish } \\
\text { colourless liquid. }\end{array}$ \\
\hline $\begin{array}{c}\text { PROPANEDIOL } \\
\text { Sigma-Aldrich, Merck Life Science S.r.l., Milano, Italy }\end{array}$ & $\begin{array}{l}\text { Propanediol is a colourless, viscous, water-soluble liquid used as an } \\
\text { antifreeze, solvent and intermediate in organic syntheses. }\end{array}$ \\
\hline $\begin{array}{c}\text { ETHYL-LACTATE } \\
\text { Sigma-Aldrich, Merck Life Science S.r.l., Milano, Italy }\end{array}$ & $\begin{array}{l}\text { Ethyl-lactate, ethyl lactate, is an ester of lactic acid and ethanol. At } \\
\text { room temperature, it appears as a colourless liquid with a fairly strong } \\
\text { odour. It is a flammable and irritating compound, it is biodegradable } \\
\text { and is called a green solvent, as it is a good solvent and is used as a } \\
\text { food additive. }\end{array}$ \\
\hline
\end{tabular}


Initially, these substances were mixed in pairs in different percentages for each: 20 and $80 \%, 40$ and $60 \%, 60$ and $40 \%$, and 80 and $20 \%$. The various compounds were mixed on slides and placed in plastic trays.

A first selection was made considering the macroscopical physico-chemical, handability and workability behaviour of the various composites, for instance:

1. corn oil is not recommended because it tends to separate from the substance with which it is mixed;

2. glycerine is a good substance that maintains a degree of viscosity that makes the compound neither too liquid nor too viscous;

3. propanediol is a good substance, very similar to glycerine;

4. ethyl-lactate has a good viscosity, but, like oil, it tends to separate a little and, moreover, it is a substance that tends to vaporize;

5. PEG 1000 is a granular substance that can be mixed with other substances with great difficulty. Nevertheless, it gives good results when mixed with propanediol or glycerine because the latter are not entirely liquid;

6. PEG 400 is an excellent substance, as it has the viscosity that makes the compound ideal; 7. PEG 200, finally, is a fairly fluid substance. It helps to obtain the desired viscosity by amalgamating with a second substance.

Hence, the compounds that have shown good characteristics and that have been chosen for the second phase of the preparation of the glass slides (mixture of the composition of the two substances with the nano-apatite based cement) are as follows: Compound n.1: 60\% glycerine, 40\% PEG 1000; Compound n.2: 40\% PEG 400, 60\% glycerine; Compound n.3: 80\% PEG 1000, 20\% propanediol; Compound n.4: 60\% PEG 1000, 40\% propanediol; Compound n.5: 80\% PEG 200, 20\% propanediol; Compound n.6: $80 \%$ glycerine, 20\% PEG 200.

These compounds were mixed with the nano-apatite cement [15-17], and a further selection of mixtures was carried out after evaluating the glass slides at 10 days. For each of the six compounds, mixtures of $66 \%, 50 \%$ and $33 \%$ of nano-apatite cement and $34 \%, 50 \%$ and $67 \%$ of the compound, respectively, were prepared.

Most of the compounds dried after 10 days at standard temperature and pressure and relative humidity of about $50 \%$. From this selection, it was decided to continue the work with a first mixture composed of $66 \%$ nano-apatite-based cement $(0.132 \mathrm{~g}), 13.6 \%$ propanediol (0.0272 mL), 20.4\% PEG 1000 (0.0408 g), herein called formulation 1, and a second mixture composed of $66 \%$ nano-apatite based cement $(0.132 \mathrm{~g}), 27.2 \%$ glycerin (0.0544 mL), 6.8\% PEG 200 (0.0136 mL), herein called formulation 2.

\subsection{Permeability Test and Analysis Cycles}

For the permeability tests, carried out by employing a Pashley permeabilimeter, model dentin discs were used to test the effectiveness of the two different composites, as performed in previous studies [20,21].

The dentin model discs were placed on $2.1 \mathrm{~cm} \times 2.1 \mathrm{~cm} \times 0.6 \mathrm{~cm}$ square centre-holed plexiglass supports with a $1.5 \mathrm{~cm}$ long metal capillary fixed in the hole. The dentin model disc was then placed so that the metal capillary is in the centre of the disc.

To measure the permeability of the model dentin disc and its variations following the application of the composite sealer sample, 20 analysis cycles per formulation were performed. These cycles are made up of four distinct phases. After each of these phases, the permeability test was performed. The infiltration of each model dental sample was evaluated by measuring the water flow in a capillary upstream of the metal one, as described in the literature [20-23].

The analysis cycle consists of four distinct phases. In the first one, once the model dentin has been attached to the plexiglass square, it is necessary to take a P400 Grit sandpaper and wet the latter with a few drops of bi-distilled water. Then, the surface of model dentin disc was rubbed on the wet sandpaper for thirty seconds, with constant cyclic movement $(10 \mathrm{~mm} / \mathrm{s})$ and load of about $50 \mathrm{~g}$. In this way, a smooth, homogeneous, 
and pure smear layer is obtained, and a thickness of model dentin of about 20-30 $\mu \mathrm{m}$ is removed. After rubbing, the model dentin is rinsed with a constant and strong 10-s jet of bi-distilled water to remove the debris, and then it is gently dabbed. Finally, the sample's permeability can be measured.

In the second phase of the cycle, the model dentin disc is subjected to the treatment with ethylenediaminetetraacetic acid (EDTA) $0.5 \mathrm{M}$ at $\mathrm{pH}$ 7.3: in practice, the surface of the model dentin is wetted with a few drops of the formulation (nano-apatite cement and liquid phase) through a microsyringe. Subsequently, for $5 \mathrm{~min}$, the surface of the model disc is brushed to obtain a uniform application of the EDTA. This acid can remove the smear layer, previously formed, and any other residue of debris and calcium, making the model dentin hyper conductive. Finally, the measurement of permeability is repeated, which is considered as the maximum opening value of the model dentinal tubules after it has been rinsed for $10 \mathrm{~s}$ with bi-distilled water.

The third phase involves the application of the formulation (cement + liquid phase), previously prepared. Since our goal is to precisely test the created sealer samples, each one is placed on the model dentin disc and repeatedly tapped with light and close strokes of a brush for $30 \mathrm{~s}$, so that the sample is distributed over the entire surface of the model dentin disc. Finally, the model dentin disc is washed under a continuous jet of bi-distilled water for $10 \mathrm{~s}$, and then the permeability test is carried out.

The last phase of the cycle consists in treating the model dentin disc with citric acid $(0.02 \mathrm{M})$ at $\mathrm{pH} 2.5$ at room temperature. A couple of drops of the latter substance are placed on the disc through a micro-syringe and tapped with firm and constant movements with a brush. Afterwards, the washing is carried out with bi-distilled water with a continuous jet for $10 \mathrm{~s}$, and then the permeability test is carried out.

Two formulations were finally selected and tested on the model dentine discs: n.1 (66\% nano-apatite-based cement (0.132 g), 13.6\% propanediol (0.0272 mL), 20.4\% PEG 1000 $(0.0408 \mathrm{~g}))$ and n.2 (66\% nano-apatite based cement $(0.132 \mathrm{~g}), 27.2 \%$ glycerin $(0.0544 \mathrm{~mL})$, 6.8\% PEG 200 (0.0136 mL).

These two final formulations were chosen because they have the best characteristics among the various prepared ones: they are compact pastes capable of making a good thread, which is a desirable feature of our work. Each model dentin disc was subjected to the four analysis phases. The permeability measurements were collected after the completion of each phase (the formation of the smear layer, the application of the EDTA, the application of the cement formulation to be tested, and finally after the acid attack). The permeability value after application of EDTA was that of maximum tubular permeability (equal to $100 \%$ ).

\subsection{SEM}

The model dentine discs treated with formulation 1 and those with formulation 2, both used in the study of permeability, were also investigated by SEM, i.e., after phase 4 (citric acid attack) of the analysis cycle. First, the discs were detached from the plexiglass base and cleaned from glue. After that, the two types of samples were placed in different containers and then in a dryer for overnight. Subsequently, three other types of samples (control samples) were specifically prepared for evaluation by scanning electron microscopy after the intermediate phases (phase 2, EDTA, and phase 3, cement formulation) of the analysis cycle:

i. model dentin control samples subjected to the formation of the smear layer and the application of EDTA;

ii. other model dentin discs after the application of the formulation 1 , which were then rinsed with bi-distilled water;

iii. other model dentin discs after the application of the formulation 2, which were then rinsed with bi-distilled water.

These last three types of samples were placed under an infrared lamp for about twenty minutes to speed up the drying process. The five types of dried samples were placed onto 
SEM metal stubs. Once dried, the samples were gold sputtered and finally observed by SEM (at a magnification of $\times 1000, \times 2000$ and $\times 3500$ ). SEM imaging and measurements were conducted considering proper instrumental setups to analyse the behaviour of the application of the two formulations, as discussed in recent literature [24].

\section{Results and Discussion}

As specified in Section 2, the two compounds selected for permeability evaluation were:

1. $66 \%$ nano-apatite based cement $(0.132 \mathrm{~g}), 13.6 \%$ propanediol $(0.0272 \mathrm{~mL}), 20.4 \%$ PEG $1000(0.0408 \mathrm{~g})$ herein called formultaion 1 , and

2. $66 \%$ nano-apatite based cement $(0.132 \mathrm{~g}), 27.2 \%$ glycerin $(0.0544 \mathrm{~mL}), 6.8 \%$ PEG 200 $(0.0136 \mathrm{~mL})$ herein called formulation 2 .

These compounds were selected based on the desired characteristics of workability and setting time. Permeability evaluation results for model dentin discs treated with the formulation 1 were reported in Figure 1. The water flows data were normalized to the maximum permeability (completely open model dentinal tubules). The model dentin discs tested for formulation 1 initially showed a permeability of about $22 \%$, due to the formation of the smear layer. Then EDTA was applied and the relative permeability of the disc was obtained (maximum permeability). With the application of the formulation 1 to the model dentin, the permeability decreased by about $80 \%$ (see Figure 1). This means that this formulation was able to effectively occlude the model dentinal tubules. Finally, after the treatment with citric acid, the permeability increased by about $12 \%$ while remaining below the maximum permeability by more than $60 \%$ (Figure 1 ). The statistical variation of 20 independent measurements is reported as a black bar on the histograms and was tested to be of about $10 \%$. The EDTA histogram has no statistical variation bar because all the other measurements (smear layer, formulation, citric acid) refer to relative permeability measurements of different starting EDTA permeability, always taken as $100 \%$.

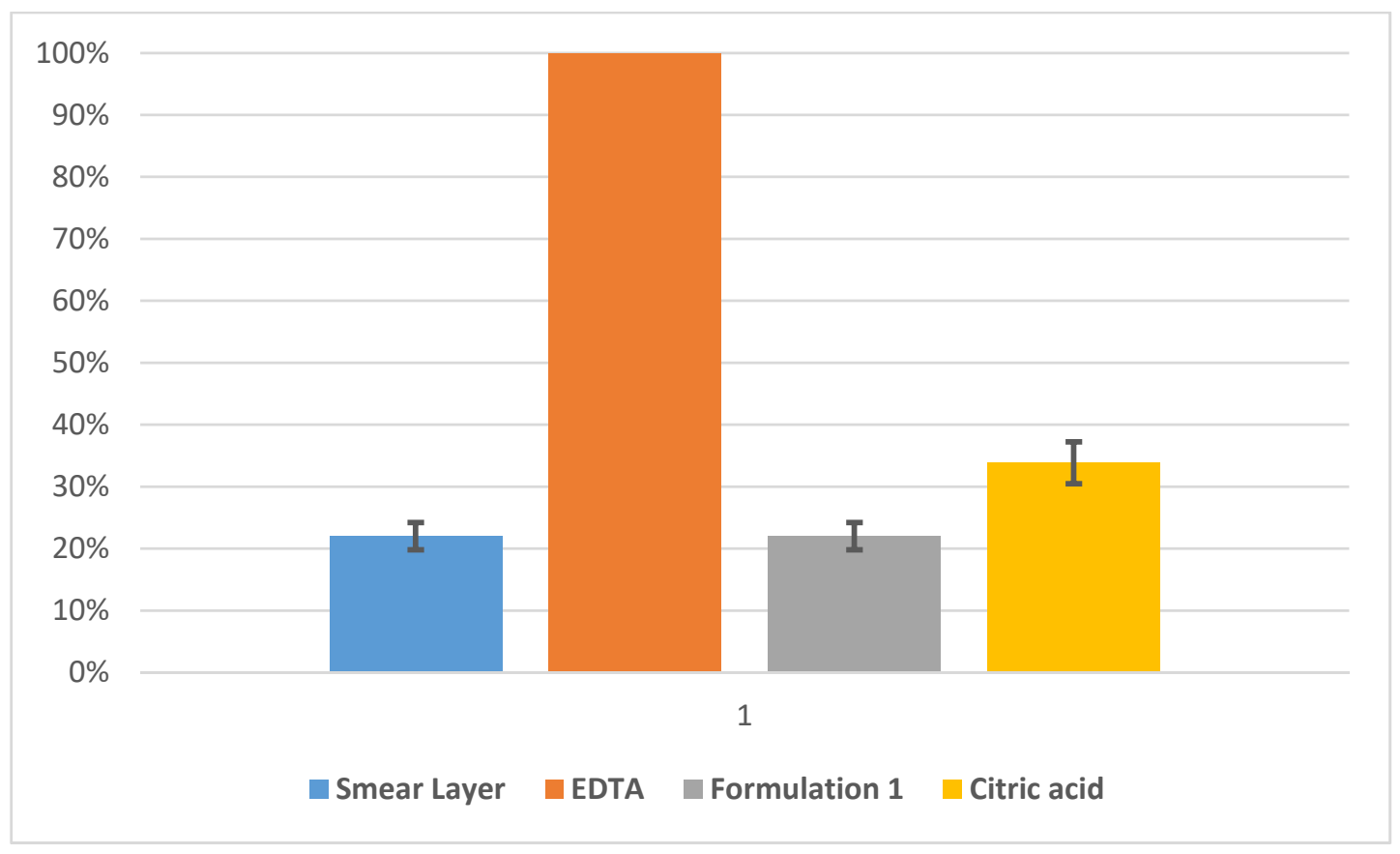

Figure 1. Normalized permeability for model dentine samples treated with formulation 1 (66\% nano-apatite based cement + $20.4 \%$ PEG $1000+13.6 \%$ propanediol) measured after the formation of the smear layer (blue), and ethylenediaminetetraacetic acid (EDTA) (orange), formulation 1 (grey) and citric acid (yellow) treatments. Statistical variation of 20 independent measurements is reported as black bars. 
Permeability evaluation results for the treatments of model dentin discs with formulation 2 are reported in Figure 2. The dentin discs employed to test formulation 2 showed a permeability value in the presence of the smear layer of about $50 \%$ lower than the maximum permeability (EDTA). The permeability measured after the application of EDTA was taken as reference value for the maximum opening of the dentinal discs, and all the other measurements were normalized to this value. With the application of formulation 2 to the model dentin, the permeability revealed a decrease of about $45 \%$ (see Figure 2 ). This means that the prepared cement was able to occlude the model tubules. Finally, the treatment with citric acid to the model dentinal discs occluded by the formulation 2 increased the permeability to about $20 \%$, while still maintaining values lower than the maximum permeability of about $22 \%$ (Figure 2). The statistical variation of 20 independent measurements is reported as a black bar on the histograms and attested to be about $10 \%$. The EDTA histogram has no statistical variation bar because all the other measurements (smear layer, formulation, citric acid) refer to relative permeability measurements of different starting EDTA permeability, always taken as $100 \%$.

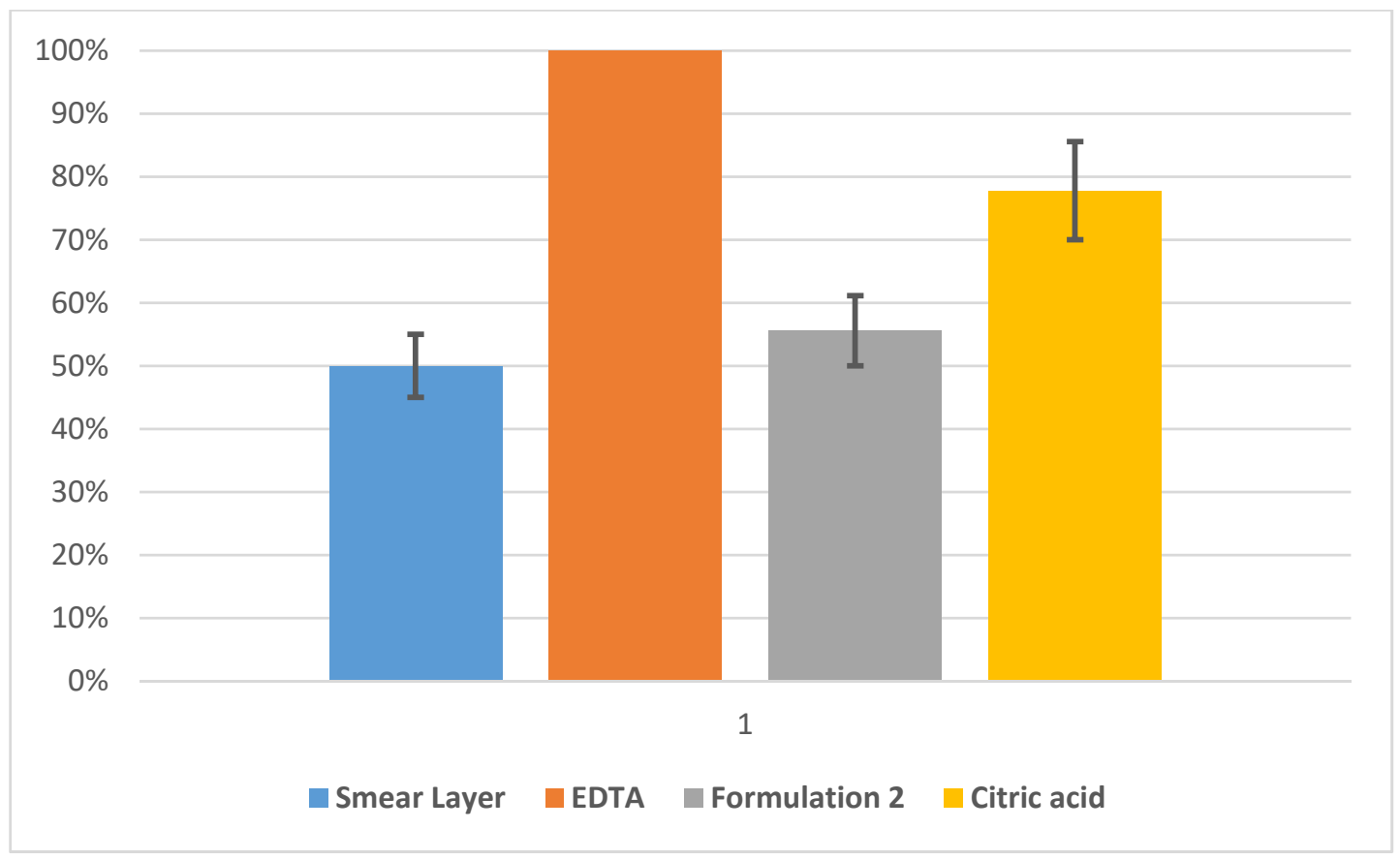

Figure 2. Normalized permeability for model dentine samples treated with formulation 2 (66\% nano-apatite based cement + $27.2 \%$ glycerin $+6.8 \%$ PEG 200) measured after the formation of the smear layer (blue) and EDTA (orange), formulation 2 (grey) and citric acid (yellow) treatments. Statistical variation of 20 independent measurements is reported as black bars.

\section{SEM Evaluation}

Control samples subjected to the formation of the smear layer and the application of EDTA showed completely open model dentinal tubules (a typical image, as an example, is shown in Figure 3).

Control samples treated with the formulation 1 showed model dentine surfaces completely covered with the cement formulation, with no evident exposed tubules (Figure 4). The formulation 1 caused the formation of large clasts (of several micrometres) and smaller ones at the surface, as evidenced in Figure 5. 


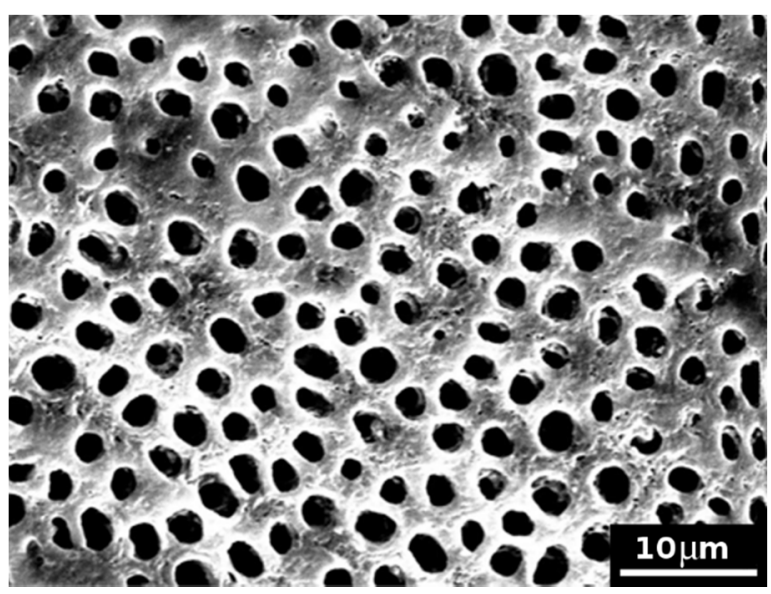

Figure 3. SEM picture $(\times 2000)$ of an untreated model dentin disc (control sample), subjected to the formation of the smear layer and subsequent application of EDTA, showing completely open model dentinal tubules.

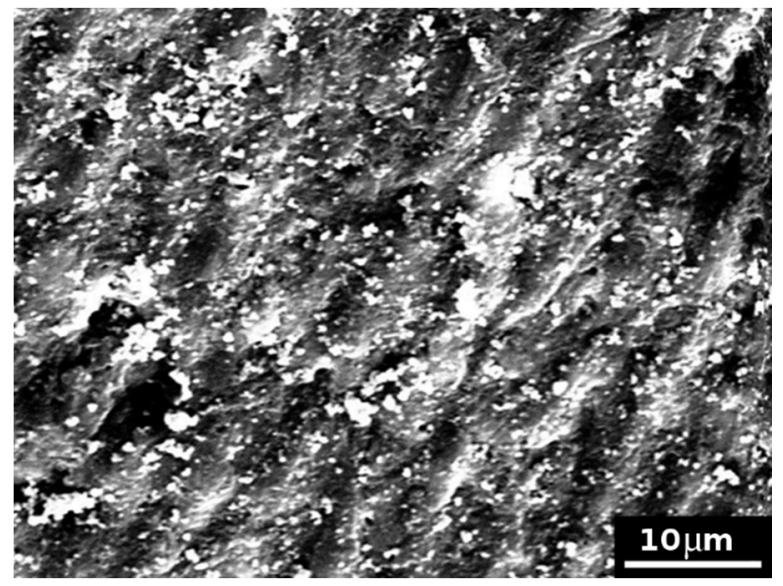

Figure 4. SEM picture $(\times 2000)$ of a model dentine surface (control sample) treated with formulation 1 with no evident exposed tubules.

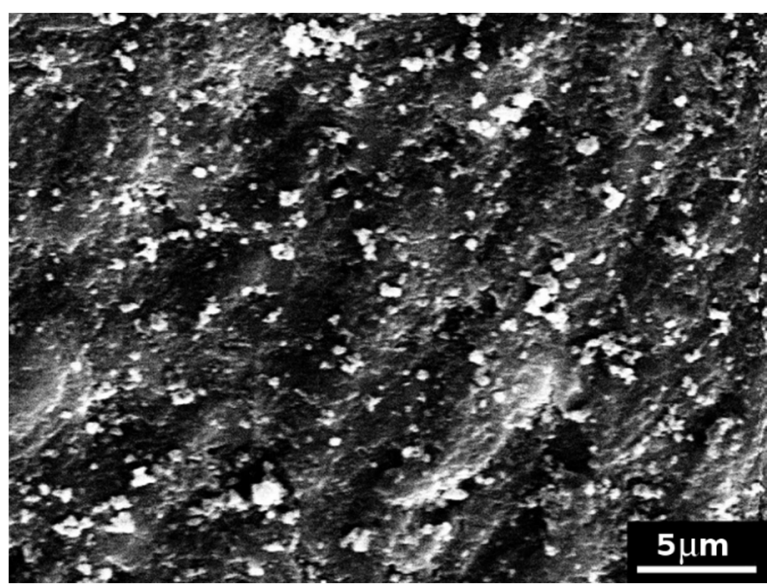

Figure 5. SEM picture $(\times 3500)$ of a model dentine disc (control sample) treated with formulation 1 , highlighting the presence of large and small clasts formed at the surface.

The formulation 1 almost completely closed the model dentinal tubules and resisted to the attack of citric acid. After the treatment with citric acid, some tubules were slightly 
opened, whereas many clasts were observed, created by the application of the cement formulation 1 and occluding the tubules (see Figure 6 as an example).

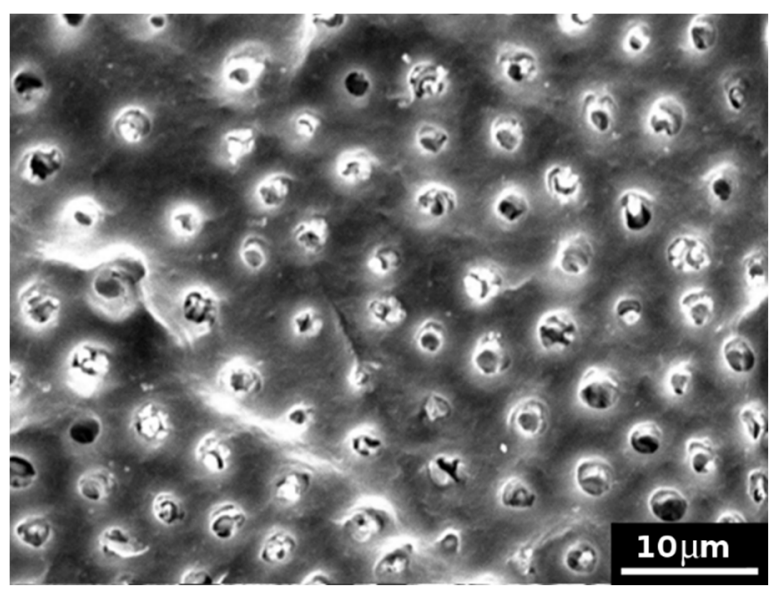

Figure 6. SEM picture $(\times 2000)$ of a model dentine surface treated with the formulation 1 and subsequently exposed to citric acid attack: model dentinal tubules appeared almost completely closed. Some tubules have been slightly opened by the acid attack, whereas many clasts are observed.

Model dentin discs subjected to the application of formulation 2 and then rinsed with bi-distilled water showed that this cement completely covered the surface of the disk (see as examples Figures 7 and 8). It is possible to observe various clasts, such as small-medium perfect crystals, several micrometres in size. Following the same protocol employed for formulation 1, other model discs were subjected to the formation of the smear layer, the application of EDTA, formulation 2 and citric acid. Figure 9 reports, as an example, an SEM micrograph acquired after citric acid attack. In comparison with Figure 7 (i.e., after the treatment with the cement formulation), where a disc surface covered with cement and tubules can be seen to be completely closed, Figure 9 showed several open tubules. These results confirm the permeability test and suggest that formulation 2 is less effective to resist citric acid attack than formulation 1 . Formulation 2 was probably not able to efficiently convey the cement inside the model tubules and remained as a layer, as shown by SEM, just onto the model dentin surface, so it was easy successively to be dissolved by the citric acid.

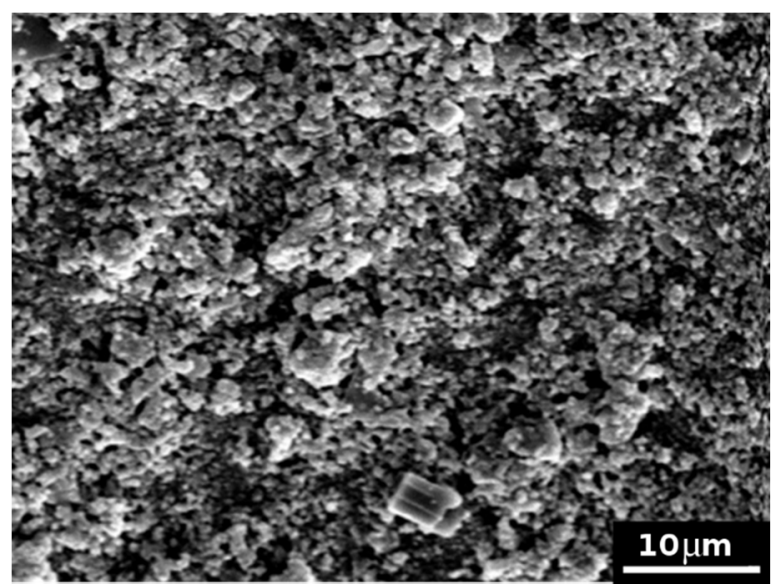

Figure 7. SEM picture $(\times 2000)$ of model dentin treated with formulation 2 showing a completely covered surface layer. 


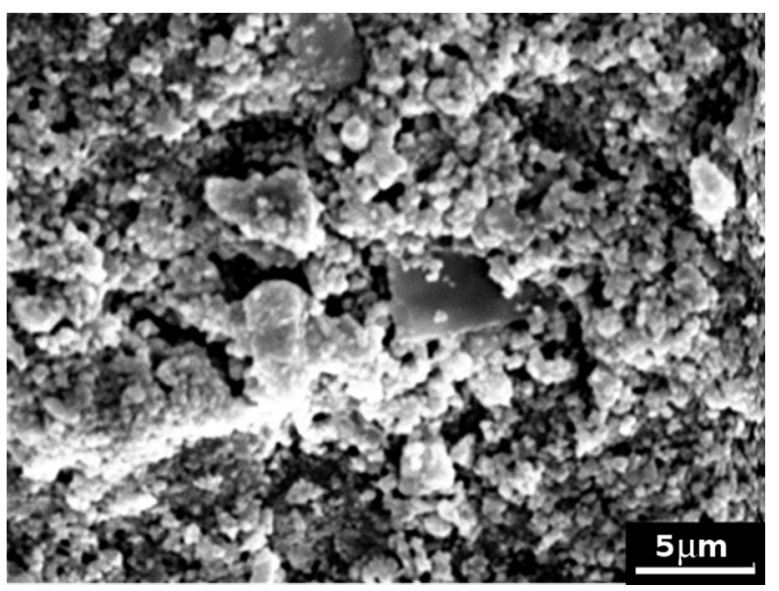

Figure 8. SEM picture $(\times 3500)$ of model dentin treated with formulation 2 showing various clasts, such as small-medium perfect crystals, several micrometres in size on the surface.

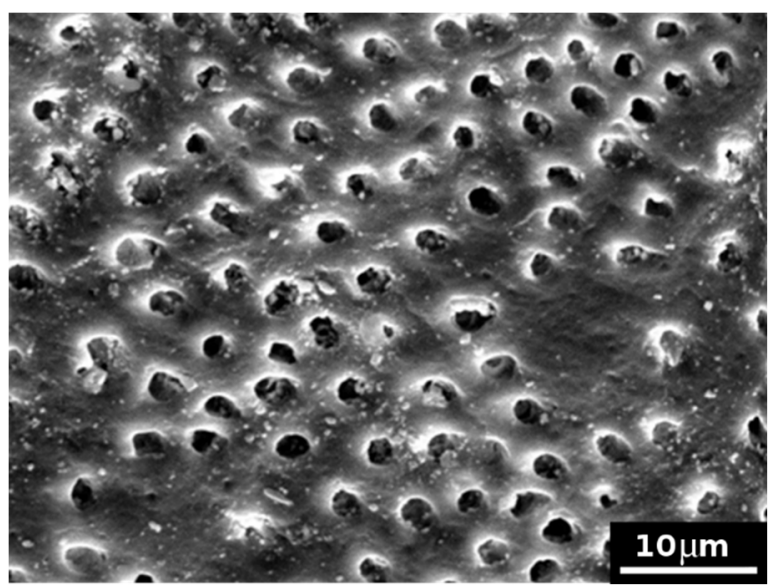

Figure 9. SEM picture $(\times 2000)$ of model dentin treated with formulation 2 and successively exposed to citric acid attack, showing several widely opened tubules.

\section{Discussion}

Endodontic sealers for non-surgical root canal treatment (NSRCT) span many compositions and attributes: zinc oxide-eugenol, salicylate, fatty acid, glass ionomer, silicone, epoxy resin, tricalcium silicate and methacrylate resin sealers. Setting time, solubility, sealing ability, antimicrobial, biocompatibility and cytotoxicity are all key aspects to the performance of endodontic sealers [25].

Bioceramic or hydraulic materials are promising bioactive candidates for hard tissue repair owing to their excellent physicochemical and biological properties.

Recently, endodontic sealers based on calcium silicates were developed due to the excellent sealing ability, biocompatibility and antibacterial properties as well as bioactivity of calcium silicate-based cements $[25,26]$. The principal limits of these cements are long setting time and difficult handling [26].

These materials are composed of silicates (dicalcium/tricalcium) or tricalcium aluminate and capable of producing hydroxyapatite when incorporated with calcium and silicon, showing functional bonding with dentine [27]. Tricalcium silicate sealers show low relative microleakage among endodontic sealers compared with silicone and resin-based cement [25].

Ready-to-use sealers consisting of only one component with a need for external water supply from, e.g., body fluid and two components sealers with internal water supply were introduced to the market. 
Both material types have the same setting reactions in common, whereby a hydration reaction of the calcium silicate is followed by a precipitation reaction of calcium phosphate [26]. Premixed, injectable sealers based on calcium silicate are hydrophilic and show good physicochemical and biological properties in vitro [28] as they are biocompatible and bioactive, features mostly attributed to the presence of calcium phosphate in their composition [29]. The target premixed calcium silicate-based sealers show results similar to or better than conventional endodontic sealers as observed in in vitro and in vivo animal studies [30,31]. Premixed cements also allow one to obtain an optimal powder-to-liquid ratio and to overcome any limitation in the workability of the material, extending the use of calcium silicate cements, which already have a good track record for endodontic applications [15].

As mentioned, hydrophilic calcium-silicate based root canal sealers, such as Totalfill BC Sealer (FKG Dentaire SA, La Chaux-de-Fonds, Switzerland), can interact with the surrounding dentinal tissue by its ability to form hydroxyapatite, thereby establishing a real connection between the filling material and dentin [29]. A strong and long-lasting link between the root canal wall and the filling is one aspect of the prevention of root canal infection, caused either by regrowth of microorganisms or newly gained infection due to coronal or apical leakage [32]. Endodontic sealers are used to seal minor discrepancies between the dentinal wall of the root canal and the root filling material, including irregularities in the apical foramen and canal [33].

Although the physical properties of calcium silicate-based sealers meet the recommendations of the International Organization for Standardization and have consistently been reported to be biocompatible, they have not overcome conventional resin-based sealers in actual practice [33]. The bioactivity of calcium silicate sealers depends on their solubility, even after setting, and this solubility might affect the quality of root canal sealing [26] despite bioceramic materials having been reported to have good stability over time [14].

In this context, the goal of the present work was to develop a new suitable "carrier" to obtain a premixed, injectable and ready-to-use bioceramic nano-apatite-based cement, exploiting the optimal biocompatibility of apatite and the sealing capability of nano-apatite crystals. For this purpose, in this preliminary investigation, the consistency and workability characteristics of some compounds that can be mixed with the nano-apatite-based cement were here analysed. The substances deemed most suitable were PEG 1000, PEG 400, PEG 200, propanediol, glycerin, corn oil and ethyl-lactate.

After an initial selection, the following two substances were completely excluded: (i) corn oil, because it has a very high tendency to separate from the mixture, and (ii) ethyllactate, because, in addition to parting from the mixture, it vaporizes at room temperature.

After the first blends, compounds were mixed with nano-apatite cement, and a further selection was made, always considering the desired characteristics (handling and setting time). This second selection criterion resulted in only two formulations suitable for the present study:

1. $66 \%$ nano-apatite based cement $(0.132 \mathrm{~g}), 13.6 \%$ propanediol $(0.0272 \mathrm{~mL}), 20.4 \%$ PEG 1000 (0.0408 g); and

2. $66 \%$ nano-apatite based cement $(0.132 \mathrm{~g}), 27.2 \%$ glycerin $(0.0544 \mathrm{~mL}), 6.8 \%$ PEG 200 (0.0136 mL).

Glycerol and PEG have been employed in calcium silicate and calcium phosphate endodontic sealer formulation $[15,34]$. Using glycerol instead of water may permit a lower liquid-to-powder ratio to obtain the same level of injectability [15]. A drawback of glycerol is that the setting time of the cement increases, because, as previously reported [15], glycerol needs to diffuse out and then be replaced by physiological fluid for setting to occur.

Because sealing ability is relevant to successful outcomes of root canal obturation, the effects of the experimental materials on model dentinal tubules occlusion were preliminarily evaluated. To test permeability and closure properties of the model dentinal tubules, the Pashley permeabilimeter was used, and scanning electron microscopy was performed to 
observe the morphology of treated model dentine surface after the experimental nanoapatite based formulations and after acid attack [20-22].

From the preliminary results obtained in this study, it was possible to state that the two synthesized composite formulations made of nanocrystals and liquid phase succeeded in occluding the model dentinal tubules: the first cement, obtained from a mixture formed of $66 \%$ nano-apatite-based cement $+20.4 \%$ PEG $1000+13.6 \%$ propanediol is managed to create a fairly full-bodied layer on the surface of the model dentin, such as to close the tubules and, moreover, managed to resist, at least to a large extent, to acid attack; the second cement, obtained from a mixture formed of $66 \%$ nano-apatite-based cement $+27.2 \%$ glycerin $+6.8 \%$ PEG 200, also managed to occlude the model dentin tubules, but showed a lower resistance to the application of citric acid. In conclusion, the preliminary experimental data here obtained indicate that the composite formulation that better satisfies the required characteristics is the one containing propanediol and PEG 1000; however, formulation 2 displayed good properties as a cement, as revealed from the reported analyses.

The results of the study indicate that it is possible to obtain, by varying the composition of the liquid phase with specific substances, an endodontic cement based on nano apatite with adequate characteristics of workability, setting and occlusion of the dentinal tubules. These results will have to be further verified considering the limitations of the present preliminary work: the effects of variations in the composition of the material on injectability, radiopacity and setting time and the sealing capacity of the material over time in association with gutta-percha will have to be determined.

Future work will be devoted to improving some aspects of the compounds reported here, such as the occlusion of the dentinal tubules and the related resistance to other acid attacks, by adding and testing other substances and optimizing the carrier/cement ratio. Further investigations are needed also to assess the clinical relevance of the relationship between bioactivity and solubility, physical properties, and biocompatibility and to develop an ideal endodontic cement that combines sealing and therapeutic effects.

\section{Conclusions}

The aim of the present work was to develop a new composite bioceramic based on calcium phosphate and a liquid phase, which could be effectively and easily used in endodontic applications (e.g., root canal filling and dentin tubules occlusion). In terms of workability, two of several nano-apatite/liquid mixtures were suitable for the desired applications. In particular, the optimal weight percentage of solid-to-liquid ratio was 66:34, with the liquid phase composed of either $13.6 \%$ propanediol and $20.4 \%$ PEG 1000 or $27.2 \%$ glycerin and $6.8 \%$ PEG 200. The occlusion capability and the acid attack resistance of these two formulations were assessed by means of permeability measurements and SEM imaging. While both composites cements successfully sealed dentinal tubules, the first cited formulation showed a better resistance against citric acid attack than the second one. These preliminary results suggest that propanediol, glycerine, PEG 200 and PEG 1000 are suitable carrier materials to realize an effective injectable nano-apatite based cement.

Author Contributions: Conceptualization, G.V., D.M., A.B. and G.U.; methodology, G.V. and D.M.; validation, G.V., D.M., A.B. and G.U.; formal analysis, G.V., D.M., A.B. and G.U.; investigation, G.V., D.M., A.B. and G.U.; data curation, G.V., D.M., A.B. and G.U.; writing—original draft preparation, G.V., D.M., A.B. and G.U.; writing-review and editing, G.V., D.M., A.B. and G.U.; visualization, G.V. and D.M.; supervision, G.V. All authors have read and agreed to the published version of the manuscript.

Funding: This research received no external funding.

Conflicts of Interest: The authors declare no conflict of interest. 


\section{References}

1. Yanpiset, K.; Banomyong, D.; Chotvorrarak, K.; Srisatjaluk, R.L. Bacterial leakage and micro-computed tomography evaluation in round-shaped canals obturated with bioceramic cone and sealer using matched single cone technique. Restor. Dent. Endod. 2018, 43, e30. [CrossRef] [PubMed]

2. Filho, M.T.; Torres, F.F.E.; Pinto, J.C.; Junior, A.O.S.; Tavares, K.I.M.C.; Tanomaru, J.M.G. Micro-computed tomographic evaluation of a new system for root canal filling using calcium silicate-based root canal sealers. Restor. Dent. Endod. 2020, 45, e34. [CrossRef] [PubMed]

3. Kopper, P.; Fiqueiredo, J.; Bona, A.D.; Vanni, J.; Bier, C.; Bopp, S. Comparative in vivo analysis of the sealing ability of three endodontic sealers in post-prepared root canals. Int. Endod. J. 2003, 36, 857-863. [CrossRef] [PubMed]

4. Jafari, F.; Jafari, S. Composition and physicochemical properties of calcium silicate-based sealers: A review article. J. Clin. Exp. Dent. 2017, 9, e1249-e1255. [CrossRef] [PubMed]

5. Haddad, A.A.; Aziz, Z.A.C.A. Bioceramic-Based Root Canal Sealers: A Review. Int. J. Biomater. 2016, $2016,9753210$.

6. Best, S.; Porter, A.; Thian, E.; Huang, J. Bioceramics: Past, present and for the future. J. Eur. Ceram. Soc. 2008, 28, 1319-1327. [CrossRef]

7. Ulian, G.; Valdrè, G.; Corno, M.; Ugliengo, P. Periodic ab initio bulk investigation of hydroxylapatite and type A carbonated apatite with both pseudopotential and all-electron basis sets for calcium atoms. Am. Mineral. 2013, 98, 410-416. [CrossRef]

8. Ulian, G.; Moro, D.; Valdrè, G. First-principles study of structural and surface properties of (001) and (010) surfaces of hydroxylapatite and carbonated hydroxylapatite. J. Appl. Crystallogr. 2016, 49, 1893-1903. [CrossRef]

9. Ulian, G.; Valdrè, G. Effect of mechanical stress on the Raman and Infrared bands of hydroxylapatite: A quantum mechanical first principle investigation. J. Mech. Behav. BioMed Mater. 2018, 77, 683-692. [CrossRef]

10. Ulian, G.; Valdrè, G. Equation of state of hexagonal hydroxylapatite (P6(3)) as obtained from density functional theory simulations. Int. J. Quantum Chem. 2018, 118, e25553. [CrossRef]

11. Parirokh, M.; Torabinejad, M. Mineral trioxide aggregate, a comprehensive literature review—Part I, chemical, physical, and antibacterial properties. J. Endod. 2010, 36, 16-27. [CrossRef] [PubMed]

12. Parirokh, M.; Torabinejad, M. Mineral trioxide aggregate, a comprehensive literature review-Part III, clinical applications, drawbacks, and mechanism of action. J. Endod. 2010, 36, 400-413. [CrossRef] [PubMed]

13. Parirokh, M.; Torabinejad, M.; Dummer, P.M.H. Mineral trioxide aggregate and other bioactive endodontic cements: An updated overview-Part I: Vital pulp therapy. Int. Endod. J. 2018, 51, 177-205. [CrossRef] [PubMed]

14. Donfrancesco, O.; Seracchiani, M.; Morese, A.; Ferri, V.; Nottola, S.A.; Relucenti, M.; Gambarini, G.; Testarelli, L. Analysis of Stability in Time of Marginal Adaptation of Endosequence Root Repair Material on Biological Samples. Dent. Hypotheses 2020, 11, 11-15.

15. Persson, C.; Engqvist, H. Premixed calcium silicate cement for endodontic applications: Injectability, setting time and radiopacity. Biomatter 2011, 1, 76-80. [CrossRef] [PubMed]

16. Lozano, F.J.R.; Bernal, D.G.; Sánchez, R.E.O.; Seltenerich, P.S.O.; Forner, L.; Moraleda, J.M. Evaluation of cytocompatibility of calcium silicate-based endodontic sealers and their effects on the biological responses of mesenchymal dental stem cells. Int. Endod. J. 2017, 50, 67-76. [CrossRef] [PubMed]

17. Dolci, G.; Mongiorgi, R.; Prati, C.; Valdrè, G. Calcium phosphates produced by physical methods in the treatment of dentin hypersensitivity. Minerva Stomatol. 1999, 48, 463-476. [PubMed]

18. Dolci, G.; Mongiorgi, R.; Prati, C.; Valdrè, G. Odontostomatologic Use of Apatite-Based Nanostructured Materials. Patent Application No. WO2000003747A2, 27 January 2000.

19. Dolci, G.; Valdrè, G.; Pilloni, A. Clinical application of a nanosized hydroxyapatite in periodontal tissue regeneration. $U \mathcal{E} U$ 2004, 2, 23-27.

20. Prati, C.; Venturi, L.; Valdrè, G.; Mongiorgi, R. Dentin Morphology and Permeability After Brushing With Different Toothpastes in the Presence and Absence of Smear Layer. J. Periodontol. 2002, 73, 183-190. [CrossRef]

21. Prati, C.; Montebugnoli, L.; Suppa, P.; Valdrè, G.; Mongiorgi, R. Permeability and Morphology of Dentin after Erosion Induced by Acidic Drinks. J. Periodontol. 2003, 74, 428-436. [CrossRef]

22. Lucchese, A.; Bertacci, A.; Zanna, S.; Chersoni, S.; Manuelli, M.; Visone, A.; Moro, D.; Valdrè, G. In vitro effects of fluoride-based and desensitizing toothpastes on dentine permeability. J. Biol. Regul. Homeost. Agents 2018, 32, 51-58. [PubMed]

23. Pashley, D.H.; Stewart, F.P.; Galloway, S.E. Effects of air-drying in vitro on human dentine permeability. Arch. Oral Biol. 1984, 29, 379-383. [CrossRef]

24. Moro, D.; Ulian, G.; Valdrè, G. SEM-EDS nanoanalysis of mineral composite materials: A Monte Carlo approach. Comp. Struct. 2020, 113227. [CrossRef]

25. Komabayashi, T.; Colmenar, D.; Cvach, N.; Bhat, A.; Primus, C.; Imai, Y. Comprehensive review of current endodontic sealers. Dent. Mater. J. 2020, 39, 703-720. [CrossRef] [PubMed]

26. Donnermeyer, D.; Bürklein, S.; Dammaschke, T.; Schäfer, E. Endodontic sealers based on calcium silicates: A systematic review. Odontology 2019, 107, 421-436. [CrossRef]

27. García, S.L.; Lloret, M.R.P.; Gironés, J.G.; Lloret, M.P.P.; Lozano, A.; Llena, C.; Rodríguez-Lozano, F.J.; Forner, L. Comparative Cytocompatibility and Mineralization Potential of Bio-C Sealer and TotalFill BC Sealer. Materials 2019, $12,3087$. 
28. Dawood, A.E.; Parashos, P.; Wong, R.H.K.; Reynolds, E.C.; Manton, D.J. Calcium silicate-based cements: Composition, properties, and clinical applications. J. Investig. Clin. Dent. 2017, 8, 12195. [CrossRef]

29. Almeida, M.M.; Rodrigues, C.T.; Matos, A.A.; Carvalho, K.K.; Silva, E.J.; Duarte, M.A.; Oliveira, R.C.; Bernardineli, N. Analysis of the physicochemical properties, cytotoxicity and volumetric changes of AH Plus, MTA Fillapex and TotalFill BC Sealer. J. Clin. Exp. Dent. 2020, 12, e1058-e1065. [CrossRef]

30. Almeida, L.H.S.; Moraes, R.R.; Morgental, R.D.; Pappen, F.G. Are Premixed Calcium Silicate-based Endodontic Sealers Comparable to Conventional Materials? A Systematic Review of In Vitro Studies. J. Endod. 2017, 43, 527-535. [CrossRef]

31. Lim, E.S.; Park, Y.B.; Kwon, Y.S.; Shon, W.J.; Lee, K.W.; Min, K.S. Physical properties and biocompatibility of an injectable calcium-silicate-based root canal sealer: In vitro and in vivo study. BMC Oral Health 2015, 15, 129. [CrossRef]

32. Donnermeyer, D.; Dornseifer, P.; Schäfer, E.; Dammaschke, T. The push-out bond strength of calcium silicate-based endodontic sealers. Head Face Med. 2018, 14, 13. [CrossRef] [PubMed]

33. Lim, M.; Jung, C.; Shin, D.H.; Cho, Y.B.; Song, M. Calcium silicate-based root canal sealers: A literature review. Restor. Dent. Endod. 2020, 45, e35. [CrossRef] [PubMed]

34. Johns, J.I.; O'Donnell, J.N.; Skrtic, D. Selected physicochemical properties of the experimental endodontic sealer. J. Mater. Sci. Mater. Med. 2010, 21, 797-805. [CrossRef] [PubMed] 